\title{
Die Auseinandersetzung zwischen Herder und Kant zum Thema der Natur der Sprache
}

DOI: http://dx.doi.org/10.12775/RF.2015.013

\section{Vorwort - die linguistische Wende in der Anthropologie der Aufklärung}

Fast immer erscheint Sprache insgesamt oder in einigen zentralen Eigenschaften als Charakteristikum des Menschen. Schon Wilhelm Humboldt hat geschrieben: "Der Mensch ist Mensch nur durch die Sprache; um die Sprache zu erfinden müsste er schon Mensch sein." ${ }^{1}$ Diese Diskussion begleiten, früher und heute, verschiedene Spekulationen über Ursprünge, Ursprachen, primitive Sprachen. Die Verschiedenheit der Sprachen, obwohl ihr Einfluß auf Meinungen und Sitten der Völker seit der Antike diskutiert wurde, erst sehr spät, deutlich erst bei Condillac, wurde auch philosphisch interessant. Der Jurist Vico dürfte der erste gewesen sein, der das Denken von der gemeinschaftsbildenden Funtion der Sprache (und die Sprache von der Imagination heroischer Individuen) her $\mathrm{zu}$ deuten versuchte. Nach ihm ist es der Theologe Herder, der bis an die Schwelle einer sprachphilosophischen Erneuerung der Ersten Philosophie gelangt. In diesem Sinne können wir ihn als Mitbegründer der aufklärischen Sprachanthropolgie apostrophieren.

Ohne auf geschichtliche und theoretische Einzelheiten einzugehen, ist es vorauszusetzen, dass in der anthropologischen Reflexion der Aufklärung der Mensch als Sprachwesen bezeichnet wurde. Ihr Programm

\footnotetext{
1 W. von Humboldt, Schriften zur Sprachphilosophie. Darmstadt 1963, S. 11.
} 
verband die Erkenntnisse der Sprachwissenschaft zu Formen und Funktionen, zur Geschichte und Entwicklung, Gemeinsamkeiten und Unterschieden menschlicher Sprachen mit dem, was über Erwerb und Lernen von Sprache in spezifischen Umgebungen und über die Grundlagen in der Gattungsgeschichte bekannt war.Viele Forscher suchten den Blick auf das, was menschliche Sprache ausmacht, mit dem kontrastierenden Blick auf tierische Kommunikationssysteme zu schärfen. Folgenreich war die dualistische Ansicht von Descartes, wonach Tieren nur ein Körper, der (physiologisch disponiert) Signale "senden” kann, nicht aber eine Seele, ein immaterieller Geist zukommt und damit Denken, Planen, Intendieren, Bewusstsein fehlen. Der menschliche Körper galt als Maschine. Paradigmatisch war der Geschtspunkt von Decartes, der in seiner Abhandlung über die Methode schreibt:,,[...] es ist ganz auffällig, daß es keinen so stumpfsinnigen und dummen Menschen gibt, nicht einmal einen verrückten ausgenommen, der nicht fähig wäre, verschiedene Worte zusammenzuordnen und daraus eine Rede aufzubauen, mit der er seine Gedanken verständlich macht; und dass es im Gegenteil kein Tier gibt, so vollkommen und glücklich es veranlagt sein mag, das ähnliches leistet. Dies liegt nicht daran, daß den Tieren Organe dazu fehlten; denn man kann beobachten, daß Spechte und Papageiern ebenso wie wir Worte hervorbringen können und daß sie dennoch nicht reden. (...) Dies zeigt nicht bloß, daß Tiere weniger Verstand haben als Menschen, sondern vielmehr, daß sie gar keinen haben. ${ }^{2}{ }^{2}$ Davon beeinflusst war auch Johann Gottfried Herder Herder ist in seiner Abhandlung über den Ursprung der Sprache (1771): „In mehr als einer Sprache hat also auch Wort und Vernunft, Begriff und Wort, Sprache und Ursache einen Namen und diese Synonymie enthält ihren ganzen genetischen Ursprung. Bei den Morgenländern ist's der gewöhnlichste Idiotismus geworden, das Anerkennen einer Sache Namengebung zu nennen: denn im Grunde der Seele sind beide Handlungen eins. Sie nennen den Menschen das redende Tier und die unvernünftigen Tiere die Stummen: der Ausdruck ist sinnlich charakteristisch und das griechische logos fasst beides. Es wird sonach die Sprache ein natürliches Organ des Verstandes, ein solcher Sinn der menschlichen Seele, wie sich die Sehkraft jener sensitiven Seele der Alten das Auge und der Instinkt der Biene seine Zelle bauet. ${ }^{\text {"3 }}$ Das Mängelwesen Mensch bedarf der Sprache, bedarf der Kultur; die Sprache entwickelt sich jenseits des tierischen Erbes (,,schon als Tier hat der Mensch Sprache $\left.{ }^{\prime 4}\right)$, dem Laute und Töne angehören, da der Mensch und nur er - die Welt reflektiert ("Besonnenheit"). Das scheint eine men-

2 R. Descartes, Abhandlung über die Methode [in:] idem, Philosophische Schriften in einem Band, Hamburg 1996, S. 94.

3 J.G., Herder, Ursprung der Sprache, Stuttgart 2006, S. 55-56.

4 Ibidem, S. 3. 
talistische Theorie der Sprache, Herder schließt das Medium allerdings an die Kommunikation an, indem er fortfährt: „Vortrefflich, daß dieser neue, selbstgemachte Sinn des Geistes gleich in seinem Ursprunge wieder ein Mittel der Verbindung ist - ich kann nicht den ersten menschlichen Gedanken denken, nicht das erste besonnene Urteil reihen, ohne daß ich in meiner Seele dialogiere oder zu dialogieren strebe; der erste menschliche Gedanke bereitet also seinem Wesen nach, mit anderen dialogieren zu können. Das erste Merkmal, was ich erfasse, ist Merkwort für mich, und Mitteilungswort für andre!"”5 Werden Gegenstände, Ideen bewusst, können sie als „Merkwörter“ kommuniziert werden. Sprache wird nicht je neu erfunden, konstituiert; in einer Kette der Bildung wird

Sprache, die aus der Reflexion kommt, über die Generationen weitergereicht, entwickelt. Diese herdersche Ideen beantworteten auf die Entscheidungsfragen der Aufklärung.

Erkenntnistheoretisch entscheidend war die Beziehung zwischen Sprache und Denken: lernt der Mensch denken erst durch die gegebene Sprache, denkt er ohne Sprache und erfindet sie nur als sekundäres Hilfsmittel? Anthropologisch entscheidend war die Frage, ob menschliche und tierische Verständigung sich wesentlich voneinander unterscheiden und damit gradueller Unterschied oder eine Wesensdiferrenz zwischen Mensch und Tier anzunehmen sei? Kosmologisch entscheidend war zugleich an dieser Frage, ob die Natur Sprünge mache oder nicht. Theologisch entscheidend war, wie, z.B. Süßmilsch aus dem gegenseitigen Begründungsverhältnis von Bernunft und Sprache gefolgert hatte, dass dann ein persönlicher Gott als Sprachmeister der ersten Menschen angenommen werden konnte, während eine mechanistische Entwicklung der Sprache aus dem Analogon der Tierschrei (Condillac, Rousseau) den propagierten Verzicht auf einen persönlichen Gott und seine Eingriffe in die Welt bekräftigten.

Herders Sprachanthropologie ging auf alle diese Entscheidungsfragen ein und antzipierte viele zeitgenösische Ideen, aber trotzdem wurde fast vergessen. Wir haben hier nicht genug Platz, um die Gründe dieser Vergessenheit zu besprechen, doch eines steht fest: der Theologe aus Riga ist bis jetzt im Schatten der kantischen Philosophie geblieben. Er war bekanntlich sein ganzes Leben lang ein vehemmenter Kritiker Kants Philosophie. Kant selbst ignorierte diese Angriffe und nur ein paar Mal nahm die Stellung zu Herders Gedanken - unter anderen in der Rezension von Ideen zur Philosophie der Geschichte der Menschheit. Es kam somit zur eigentlichen Auseinandersetzung zwischen beiden Philosophen gar nicht. Die wichtigsten polemischen Argemente beinhaltet Herders Abhandlung Metakritik der Kritik der reinen Vernunft, die doch keinen Widerhall bei Kant gefunden hat. Das soll uns nicht wundern,

\footnotetext{
5 Ibidem, S. 56.
} 
weil wir mit zwei ganz verschieden Paradigmen der Philosophie zu tun haben. Einerseits systematisches transzendentales System mit apriorischen Kategorien und andererseits eher rapsodische, skizzenhafte Gedankengänge, die ganz auf die systematischen Ansprüchen verzichten. Es scheint, als ob es kein tertium comparationis, keine Gemeinsamkeit zwischen zu vergleichender Ansätze gäbe. Kant interessiert sich vor allem für die Letztbegründung der apriorischen Gesetze menschlicher Vernunft und Herder präsentiert empiristische Auffasung, die die Existenz apriorischer Formen der Erkentnnis völlig ablehnt. Es scheint, als ob ein unüberbrückbaren Abgrund sich vor ihnen auftat. Aus gegenwärtiger Perspektive, besonders vom Standpunkt heutiger Anthropologie und Sprachphilosophie können wir einige Herders Postulate und Vorwürfe richtig einschätzen. Trotz aller Unterschiede können wir auf einen gemeinsamen Nenner hinweisen, nämlich auf das Problem der Sprache. Herder wirft Kant die Sprachvergessenheit vor. Kant reagierte nich darauf - wahrscheinlich deswegen, dass er an seinem System der kritischen Philosophie intensiv arbeitete und Herders Polemik ganz unterschätzte. Im vorliegenden Vortrag stelle ich seine Auffasung der Sprache dar, dann versuche ich, die wichtigsten Herders Thesen zu diesem Thema zu rekonstruieren.

\section{Die Sprache bei Immanuel Kant}

Es findet sich bei Kant keine ausgearbeitet, zusammenhängende Sprachphilosophie. Dennoch dürfte kein Philosoph der Neuzeit eine größere Wirkung auf die Philosophie der Sprache gehabt haben als er. Dies steht in einem engen Zusammenhang mit der Wendung zur Transzendentalphilosophie, die sich nicht direkt auf Gegenstände, sondern auf Bedingungen der Möglichkeit von Gegenständen richtet. Zu solchen Bedingungen gehört nach Kant, wie zu zeigen sein wird, durchaus auch die Sprache, aber eben nich als Gegenstand der Betrachtung, sondern als Bedingung der Möglichkeit von Gegenständen.

Durch die Konzentration der Kantrezeption auf die Kritik der reinen Vernunft und deren thematische Ausrichtung auf reine Verstanesbegriffe lag die Bedeutung der Sprache mit ihren "gegebenen” Begriffen nicht primär im Blick. Die „Kritik” ist für Kant aber eigentlich nur eine Vorarbeit für die Philosophie, und die Kritik der Urteilskraft als letzte „Kritik” bringt ausdrücklich in Erinnerung, dass „die Gegenstände der empirischen Erkenntniß, außer jener formalen Zeitbedingung", unter der sie als Gegenstände der empirischen Erkenntnis überhaupt stehen „noch auf mancherlei Art bestimmt oder, soviel man a priori urtheilen kann, bestimmbar" sind, „,so daß specifisch-verschieden Naturen außer dem, was sie als zur Natur überhaupt gehörig gemein haben, noch auf un- 
endlich mannigfaltige Weise Ursachen sein können." ${ }^{6}$ Wenn dazu die Sprache beiträgt, kann das allerdings nicht die Sprache sein, insofern sie selbst Gegenstand (z.B. in einer wissenschaftlichen Sprachbetrachtung) ist, sonder nur die Sprache in ihrem jeweiligen subjetiven Gebrauch. Es kann sich deshalb nicht a priori bestimmen lassen, wie sie sich auf das Denken und Handeln auswirkt.

Sprache ist für Kant primär ein empirisches Bezeichnungsvermögen, das als solches nicht genügend ist, hinreichend das reine Denken zu begründen. Kant gibt doch zu, dass die Sprache in ihrer formalen Struktur einen Hinweis auf die kategoriale Struktur des Denkens geben kann, aber zugleich behauptet er, es sei unmöglich, von der Analyse der Sprache das vollständige System der Kategorien deduzieren. Kant weiß, daß in jeder empirischen Sprache eine anonyme Form der Weltinterpretation funktioniert und sie aller philosophischen Reflexion vorhergeht. Das wird deutlich nich nur an ihren grammatischen Formen, sondern auch sozusagen an ihrer metaphorischen Potenz. Im $\S 59$ der „Kritik der Urteilskraft” betont Kant, daß die Sprache „unzählige . . . nicht schematische, sondern symbolische Hypo-typosen, und Ausdrücke für Begriffe nicht vermittelst einer direkten Anschauung, sondern nur nach einer Analogie derselben"7 enthalte. Die Hypotypose ist eine Darstellung, die die empirischen Begriffe versinnlicht. Als solche kann sie schematisch oder symbolisch werden. Wenn einem Verstandesbegriff die korrespondierende Anschauung a priori gegeben wird, haben wir dann mit der schematischen, direkten Hypotypose (also Darstellung) zu tun. Die symbolische Darstellung des Begriffs ist immer indirekt, sie bedient sich der Analogien, also auch empirischer Anschaunngen. Weil Sprache keine schematischen „Hypotyposen” enthält, genügt sie nicht den Ansprüchen einer transzendentalen Methode. Als Symbolisierungsleistung besteht sie vielmehr aus freien Produkten der Einbildungskraft, die jedoch nicht auf Wesensaussagen abzielen, sondern Gegenstände in Analogie zu anderen Vorstellungen versinnlichen, bildhaft machen. Die Symbolisierungsleistung der Sprache bedient sich im Gegensatz zur reinen transzendentalen Technik empirischer Anschauungen. Aus diesem Grunde kann Kant zwar feststellen, daß die Sprache Wirklichkeit symbolisch erschließt und in dieser Funktion durch keine andere Instanz ersetzt werden kann. Aber weil die Sprache als empirische Technik auf das Verfahren der bildhaften Vergegenwärtigung ebenso angewiesen ist, kann eine transzendentale Deduktion der Kategorien nicht ersetzen.

6 I. Kant, Kritik der Urteilskraft, Hamburg 2002, S. 91.

7 Ibidem, S. 296. 
Im Normalfall werden in der Sprache geistige Empfindungen „[...] in Schattenbilder der sinnlichen Dinge umgeschaffen." ${ }^{8}$ Zentrale metaphysische Begriffe, enthalten deshalb für ihn „eine Zweideutigkeit”, daran die Einschränkung der Sprache schuld ist. Als Ursache dieser „Zweideutigkeit" betont Kant die Tatsache, daß die Bedeutung der Worte aus dem gewohnten „Redegebrauch" resultiert. ${ }^{9}$ Deshalb ist notwendig eine Analyse und mögliche Korrektur zentraler metaphysischer Begriffe, die vom empirischen Redegebrauch der Umgangssprache und von ihrer eigenen Tradition unabhängig sein sollte. Es geht in der Metaphysik darum, daß sich der Mensch nach gesicherten und deshalb intersubjektiv gültigen Kriterien in der Welt „orientiert”. Die Quelle der Irritationen in dieser fundamentalen Orientierungsleistung der menschlichen Subjektivität ist für Kant der Einfluß der Sinnlichkeit auf Verstand und Vernunft. Das Zentrum des transzendentalphilosophischen Ansatzes bildet deshalb die Deduktion der Kategorien. Aus der empirisch bestimmten Sprache aber können die Kategorien des reinen Verstandes allenfalls in einem „rhapsodischen” Verfahren, d. h. zufällig aufgezählt, aber nicht in systematischer Vollständigkeit deduziert werden. ${ }^{10}$

Der Sprachbegriff Kants, so wie er über den der Metaphysik hinausweist hinausweist, entzieht sih einer "Sprachphilosophie", verstanden als Philosophie über einen abgrenzbaren Gegenstandsbereich. Der Sprechende hört sich im Sprechen unmittelbar selbst sprechen. Nur dadurch, dass er sein Denken unmittelbar auf ",sich" zurückbezieht und darin „, für such selbst” ein „anderer” ist, kann er überhaupt etwas zum Gegenstand haben. Die hier "deiktisch" genannte Komponente gehört unaufhebbar zur Sprache als solcher un damit auch zum Denken als einem „Reden mit sich selbst."11

Deshalb kann "die Sprache” auch nur in einer Anthropologie in pragmatischer Hinsicht zur Sprache kommen. Kant geht es hier darum, über die „Vermögen” des Menschen nicht mehr transzendentalphilosophisch, sonern pragmatisch zu sprechen, und zwar unter dem Gesichtspunkt, dass man um der Orientierung im Handeln willen jewiels mit der Be-

8 I. Kant, Träume eines Geistersehers, erläutert durch Träume der Metaphysik [in:] Kant's Gesammelte Schriften „Akademieausgabe“, Königlich Preußische Akademie der Wissenschaften, B. 2, Berlin 1912, S. 339.

9 I. Kant, Untersuchung über die Deutlichkeit der Grundsätze der natürlichen Theologie und der Moral [in:] Kant's Gesammelte Schriften "Akademieausgabe“, Königlich Preußische Akademie der Wissenschaften, B. 2, Berlin 1912, S. 284.

10 I. Kant, Prolegomena zu einer jeden künftigen Metaphysik, die als Wissenschaft wird auftreten können [in:] Kant's Gesammelte Schriften „Akademieausgabe“, Königlich Preußische Akademie der Wissenschaften, B. 4, Berlin 1911, S. 322-326.

11 I. Kant, Anthropologie in pragmatischer Hinsicht [in:] Kant's Gesammelte Schriften "Akademieausgabe“, Königlich Preußische Akademie der Wissenschaften, B. 7, Berlin 1917, S. 192. 
stimmung, „was" etwa und damit dann auch, „was" der Mensch und „was" seine Sprache sei, pragmatisch zu Ende kommen muss und dann, je nachdem, was "dabei im Spiele ist"12, das Ansehen als bestimmt für die Sache nimmt und diese Ansicht „für wahr" hält.

In der Anthropologie wird die Sprache „in pragmatischer Hinsicht” unter dem Aspekt des „Bezeichnungvermögen” behandelt. Das „Bezeichnungsvermögen” ist „das Vermögen der Erkenntnis des Gegenwärtigen als Mittel der Verknüpfung der Vorstellung des Vorhergesehenen mit der des Vergangenen”, und die "Handlung des Gemühs [,] diese Vernüpfung zu bewirken [,] ist die Bezeichnung." ${ }^{13}$ In diesem Bezeichnungsvermögen geht also nicht darum, Erkanntes nachträglich zu bezeichnen, sondern um eine "Verknüpfung" der Vorstellung des Vorhergesehenen über das Gegenwärtige hinweg mit der Vorstellung des Vergangenen. Das Gegenwärtige wird im Bezeichnen als „Mittel” für diese „Verknüpfung: über die Zeit hinweg erkannt, und dadurch esnur als "Mittel” genommen und als es selbst gar nicht erst bewusst vorgestellt wird, gelingt die Verknüpfung der zeitlich getrennten Vorstellunegn. Nur dadurch kommte es zu einer zusammenfassenden Vorstellung eines „bleibenden” Zusammenhang von „etwas” über die Zeit hinweg. Als an ihm selbst Übergangenes ist das "Gegenwärtige" unmitelbar erkanntes Zeichen für "etwas”, das unmöglich selsbt gegeben sein kann, weil es als „Dauer" gedacht ist, die von der Vergangenheit über die Gegewart, in dr allein etwas "gegeben” sein kann, hinweg in die Zukunft hinausreicht. Das Gegenwärtige ist als unmittelbar Übergangenes Zeichen für die umfassende Einheit der Vorstellung, die selbst anders nicht "gegeben" is. Es hat deshalb mit dem, was sich in ihm vergegenwärtigt, nichts gemeinsam und ist damit in dieser Zeitstruktur ",charakteristisches" Zeichen.

Das „Bezeichnungsvermögen” ist nichts anderes als die Einheit der Apperzeption, aber unter dem (anthropologisch-pragmatischen) Aspekt, dass das Subjekt um des Handelns willen den Prozess der Begriffserklärung abbrechen und zur Bildung eines Urteils kommen muss, ohne dass eine "adäquate" Referenz auf eine transzendente "Sache selbst" dieses Ende bestimmen könnte.

Kant leugnet nicht, daß in der Sprache immer schon ein Verstehen von Wirklichkeit

geleistet ist und daß dieses Wirklichkeitsverständnis im alltäglichen Redegebrauch mit hinreichender Präzision „funktioniert”. Die apriorischen Kategorien und Prinzipien sind völlig von der Sprache unabhängig. Insobesondere in der Kritik der reinen Vernunft wird der Sprache nur eine marginale Rolle zugewiesen. Die Frage, die Kant nicht unmittelbar

12 I. Kant, Kritik der reinen Vernunft, Hamburg 1967, S. 743 (B 853).

13 I. Kant, Anthropologie ..., op. cit., S. 191. 
stellt, nähmlich nach der Sprache, ihrem Sinn und ihrer Bedutung, hat natürlich mit der transzendentalen Methode zu tun. Allegemein gesagt scheint seine Transzendentalphilosophie aufgrund ihres übergeschichtlichen Begriffs der Vernunft mit einer Philosophie der Sprache nicht vereinbar zu sein. Kant beharrt letztendlich auf den Dualismus von Denken und Sprache. Begriffe, Gedanken und Vorstellungen sind ontologisch vorsprachlich. Diesbezüglich ist das sprachliche Zeichen nur ein Verbindungsmedium, welches die Vorstellung und den Gegenstand verknüpft und die intersubjektive Kommunikation gewährleistet. Inhaltlich tragen die sprachlichen Zeichen nichts bei. Sie sind für die objektive Erkenntnis nichts Bedeutendes. Kant glaubt zudem, die Gedanken sind in der intersubjektiven Kommunikation identisch.

\section{Herders Kritik der Vergessenheit der Sprache bei Kant}

Wie schon erwähnt wurde, Herder als Philosoph hat bis jetzt im Schatten der kantischen Philosophie geblieben. In seiner berühmt gewordenen Rezension des ersten und des zweiten Teils der Ideen zur Geschichte der Menschheit von 1785 stellt Kant die Frage, ,[...] ob nicht statt nachbarlicher Übergänge aus dem Gebiete der philosophischen in den Bezirk der poetischen Sprache, zuweilen die Grenzen und Beziehungen von beiden völlig verrückt sein." ${ }^{14}$ Der Vorwurf Kants „logische Pünktlichkeit der Begriffe” vermissen zu lassen ebenso wie „sorgfältige Unterscheidung und Bewährung der Grundsätze" und eben dadurch philosophische und poetische Sprache zu vermischen als ein dilettantischer Philosoph, musste auf Herder verletzend wirken. Und er war tief gekränkt.

Herders Reaktion auf diese Kritik kommt am deutlichsten in seiner Metakritik der Kritik der reinen Vernunft, wo er von Kant behauptet: „Er konstituiert in Wortlarven die Übervernunft, die alle Philosophie abschneidet." 15 Solche rethorischen Schlussfolgerungen machten es unmöglich, sein Anliegen auch nur wahrzunehmen, diese seine ernstzunehmende Fragen: „Was ist Verstand und Vernunft? Wie kommen sie zu ihren Begriffen? Wie knüpfen sich solche? Was für Recht haben wir,

14 I. Kant, Recensionen von J.G.Herders Ideen zur Philosophie der Geschichte der Menschheit. Theil 1. 2. [in:] Kant's Gesammelte Schriften "Akademieausgabe“, Königlich Preußische Akademie der Wissenschaften, B. 8, Berlin 1923, S. 45.

15 J.G. Herder, Eine Metakritik zur Kritik der reinen Vernunft [in:] „Schriften zur Literatur und Philosophie, 1792-1800" (hrS. von H.D. Irmscher), Frankfurt am Main 1998, B. 8, S. 343. 
uns einige derselben allgemein und notwendig zu denken."16 Viele alte und jüngste Interpreten haben Herder vorgeworfen, dass er in erster Linie den Verfasser der Kritik der reinen Vernunft und nicht nur sein Werk kritisieren wollte. Ich werde darauf in meinem Vortrag nicht eingehen, ich konzentriere mich eher auf der Erklärung des Grundunterschiedes zwischen Kant und Herder in Bezug auf die Sprache.

Kant betrachtet Sprache als „Bezeichnung der Gedanken” als Mittel "sich selbst und andere zu verstehen", daraus folgt doch - wie Herder meint -, dass ohne Sprache das Denken überhaupt nicht möglih sei. Die Gedanken sind sonst durchaus nicht frei von der Sprache. Das stetzt aber eine urspüngliche Einheit von Denken und Sprache voraus, die Kant mit den traditionellen Kategorien der Vernunft nich errklären kann. Die Bedeutung der Sprache für die Vernunft - in Herders Terminologie - für die menschliche Seele - ist entscheidend. Die Vernunft denkt nicht nur und ordnet ihre Gedanken mit Worten, sondern „,[..] sie bezeichnet sich selbst auch mittelst der Sprache."17 Wenn Herder auf die Griechen zurücksieht, die "Vernunft und Rede mit einem Wort logos bezeichneten"18, so ist für ihn der Ausgangspunkt, die Sprache der jewieligen Nation als „Organon unserer Vernunft"19 anzusehen. Viele Mißverständnisse und Widersprüche liegen nach Herder nur an dem von der Vernunft schlecht gebrauchtes Werkzeug der Sprache.

Bekanntlich es gibt für Kant zwei Stämme der menschlichen Erkenntnis: Sinnlichkeit und Verstand. „Begriffe gründen sich also auf der Spontaneität des Denkens, wie sinnliche Anschauungen auf der Rezeptivität der Eindrücke. Von diesen Begriffen kann der Verstand keinen anderen Gebrauch machen, als dass er urteilt. “20 Für Herder beruht alle Erkenntnis auf der Grundlage der sinnlichen Erfahrung und unterliegt der geschichtlichen Entwicklung. Unverständlich ist für ihn Kants Auffassung, dass der Verstand ein aktives Vermögen hat, Begriffe zu bilden. Alle Begriffe werden doch durch sinnliche Einbildungkraft geformt, es gibt keine reinen Kategorien, die nicht durch Sinne vermittelt würden. Kurzgesagt Vernunft ist sinnlich, gechichtlich und sozusagen versprachlicht

Nach Herder Kant ist zum Opfer dem szientifischen Bewußtsein gefallen, das auf der Illusion der totalen Vorurteilslosigkeit beruht. Herders Skepsis richtet sich gegen die Möglichkeit einer voraussetzungslosen theoretischen Letztbegründung menschlichen Denkens und Handelns. Jede Begründung wird immer in die Sprache und Affekte verwickelt, die

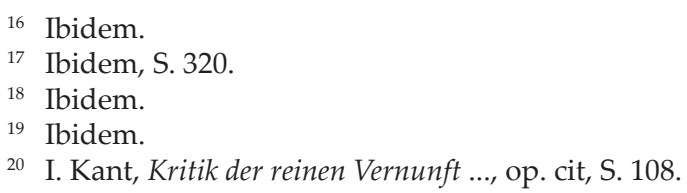


ihrerseits von der wechselnden sinnlichen, und historisch geprägten Erfahrung abhängt. Der abstrakte Anspruch auf Allgemeinheit, der in dem neuzeitlichen Wirklichkeitsverständnis vorzuherrschen beginnt, orientiert sich an der mechanisch konzipierten Physik und führt nach Herder dazu, daß die konkreten Lebensprobleme des Menschen gar nicht mehr verstanden werden können.

Der Philosoph kritisiert den rationalistischen Sprachbegriff des 18. Jahrhunderts, der Sprache als technisches Instrumentarium des Denkens auffaßt. aus diesem Verfahren resultiert ein Verlust des dem Menschen unverzichtbaren Anspruchs auf Anschaulichkeit seiner Erkenntnisse, weil die Fachsprache der Schulphilosophie lediglich auf der „Wirkung der Abstraktion" beruht. Indem sie im Abstraktionsvorgang die natürlich-sinnliche Wirklichkeit ausschaltet, konstruiert sie lediglich einen "Scheinbegriff", dessen Bedeutung intersubjektiv nicht mehr verstanden werden kann. ${ }^{21}$

Das Motiv dieser Sprachkritik ist keine radikale Sprachskepsis. Angesichts der Erstarrung der Sprache zu einem der sinnlichen Fülle der Natur gegenüber autonomen Begriffsgerüst verweist Herder vielmehr auf die in allen Sprachen vorfindbare produktive Kraft. Er expliziert sie zunächst am Problem des Sprachursprungs. Dieses Thema behandelt er primär deshalb, weil er die grundsätzliche energetische Funktion der Sprache bewußt machen will, die über ihrem alltäglichen Gebrauch als eines bloßen Kommunikationsinstruments vergessen wird.

Nach Herder ist der Ursprung der Sprache und ihrer ersten Begriffe eine freie Kraftkonzentration der menschlichen Seele, in der sie ihre Grundkraft betätigt. ${ }^{22}$ Aus diesem Akt heraus sondert sich der „innere Sinn”, ,ein Merkmal" ${ }^{23}$ ab und formuliert im Zustand höchster Kraftanspannung ein „innerliches Merkwort” als ein "gefaßtes Zeichen." ${ }^{24}$ Als Grund der Sprachentstehung betrachtet Herder nicht mehr, wie im 18. Jahrhundert weitgehend akzeptiert worden war, das Urteil einen Akt der Synthesis der in reduktiver Analyse gewonnenen einfachen Ideen, sondern ein Energiepotential, das den menschlichen Verstand an das überlegene Potential naturhafter Energie bindet. Das System der Sprache hat deshalb für Herder nicht mehr wie noch bei Kant als intellektuelle Methode die differenziertesten Operationen des menschlichen Verstandes zu ermöglichen. Sie hat auch nicht mehr das Ziel, die verwirrende Vielfalt der Natur durch ein Gefüge begrifflicher Fixierungen zu neutra-

21 J.G. Herder, Wie klebt in der Weltweisheit der Gedanke am Ausdruck, sinnlich, technisch oder grammatisch? [in:] Herders Sämmtliche Werke. (hrs. von B. Suphan), 1. Band, Berlin 1877, S. 418, 415.

22 J.G. Herder, Abhandlung über den Ursprung der Sprache, Stuttgart 1986, S. 32, 71.

23 Ibidem, S. 41.

24 Ibidem, S. 42. 
lisieren. Vielmehr soll sie in einer Fülle prägnanter Bilder aufgrund ihrer energetischen Potenz Wirklichkeit in ihrer sinnlichen Konkretheit und in ihrer Lebendigkeit dem menschlichen Bewußtsein erschließen. Auf dieser Funktion beruht ihr essentiell metaphorischer Charakter. Herderveranschaulicht diese Intention der Sprache durch eine Umkehrung der Metapher der "nackten Wahrheit” im Sinne der neuzeitlichen Wissenschaftsvernunft. Während das Streben der Wissenschaft nach „nackter Wahrheit" gerade verhindert, daß Welt in ihrer sinnlichen Fülle dem verstehenden Subjekt anschaulich zugänglich wird ${ }^{25}$, ist für Herder die Sprache das unerläßliche „Kleid der Wahrheit”. Es soll die Natur nicht verbergen, sondern gerade so viel von ihr enthüllen, daß ihre sinnlichen Reize ,,[...] durch die Sprache, wie der Busen der Phryne durch einen seidenen Nebel, durch das Wassergewand der alten Statuen, das sich an die Haut anschmiegt, durchschimmern." ${ }^{26}$ Auf diese Weise wird Natur nicht auf ein mathematisch beschreibbares Gerüst reduziert, sondern bleibt in ihrer sinnlichen Konkretheit gegenwärtig.

Entscheidend ist, daß die Sprache aufgrund ihrer metaphorischen Potenz auf ein dem Menschen eigenes und der Natur analoges energetisches Potential zurückverweist. Dieses wird nicht bemerkbar in der enthüllenden Offenheit des Lichts, sondern hat seinen Ort in der „dunkle(n) Gegend der menschlichen Seele." ${ }^{27}$ In ihr herrscht nicht lumen naturale - die Klarheit logischer Ordnung, sondern sie ist, vom Standpunkt der schulphilosophischen Logik aus gesehen, der unordentliche Ort der unteren Erkenntnisvermögen, „wo sich die Begriffe durchkreuzen und verwickeln". Für Herder aber ist dieser Ort die Quelle produktiver Tätigkeit, die sich primär im Erzeugen sprachlicher Bilder ausweist.

Ausgangspunkt der menschlichen Erkenntnis bilden nicht die einfachen Ideen, sondern die ganz elementaren sprachlichen Bilder, die in ihrer sinnlichen Fülle einen steten Bezug des menschlichen Bewußtseins zu der Natur garantieren. Der Gegenstand der Erkenntnis ist ausschließlich im sprachlichen Bild erfahrbar. ${ }^{28}$ Die „inneren Worte”, die in diesen Bildern zum Ausdruck kommen, übernehmen genau die Funktion, die innerhalb der Schulphilosophie den einfachen Ideen zugekommen war. Nach Herder wird Seiendes als sinnlich wahrnehmbare Einheit erfahren, nicht aber als eine diffuse, heterogene Vielfalt sinnlicher Impressionen,

25 Por. H. Blumenberg, Paradigmen zur Metaphorologie, Bonn 1960, S. 54.

26 J.G. Herder, Von der Lebensarten der Sprache [in:] Herders Sämmtliche Werke. (hrs. von B. Suphan), 1. Band, Berlin 1877, S. 162.

27 J.G. Herder, Abhandlung..., op. cit., S. 87

${ }_{28}$ Für eine philosophisch fundierte Explikation dieses Satzes in der gegenwärtigen Diskussion vgl. H.-G. Gadamer, Wahrheit und Methode, Tübingen, 1965, S. 450: "Sein, das Verstanden werden kann, ist Sprache". 
die erst durch ein bestimmtes Ordnungssystem des Verstandes dem menschlichen Bewußtsein zugänglich werden.

\section{Anthropologisches Fundament der Sprachphilosophie}

Herder gründet seine philosophische Konzeption auf ein anthropologisches Fundament. Dabei fragt er ausschließlich nach der natürlichen Organausstattung des Menschen. Aus ihr resultiert für ihn dessen Stellung im Zusammenhang der Natur und Geschichte. Herder hat die Frage nach der natürlichen Leistungsfähigkeit der menschlichen Vernunft in der Preisschrift über den Sprachursprung durch die qualitative Unterscheidung zwischen Mensch und Tier beantwortet. Das Tier lebt ganz innerhalb eines durch Instinktreaktionen kurzgeschlossenen Handlungskreises: „Jedes Tier hat seinen Kreis, in den es von der Geburt an gehört, gleich eintritt, in dem es lebenslang bleibet, und stirbt: nun ist es aber sonderbar, daß je schärfer die Sinne der Tiere und je wunderbarer ihre Kunstwerke sind, desto kleiner ist ihr Kreis: desto einartiger ist ihr Kunstwerk." ${ }^{29}$ Der Mensch hat dagegen ,[...] keine so einförmige und enge Sphäre, wo nur eine Arbeit auf ihn warte." ${ }^{30}$ Vom Tier unterscheidet sich der Mensch nicht nur quantitativ durch die Erweiterung des tierischen Handlungskreises, sondern mit ,[...] dem Menschen ändert sich die Szene ganz." ${ }^{31}$ Der Mensch ist ein ganz besonderes Wesen, ein Mängelwesen. Als Tier wird er an keine Umwelt angepasst, er hat keine spezialisierte Instinkte und sinnliche Organe, er ist in diesem Sinne ganz weltoffen. Diese ungünstige Anfangssituation hat jedoch einen zweckmäßigen Sinn. Die menschliche Unfertigeit wird kompensiert durch die Sprache, die vor dem Menschen eine einmalige im tierischen Reich, fast unbegrenzte Perspektive der Freiheit eröffnet. Was interessant ist, den ähnlichen Ausgangspunkt in der Beschreibung der menschlichen Entwicklung nimmt auch Kant in seiner Schrift Idee zu einer allgemeinen Geschichte in weltbürgerlicher Absicht an. Für Kant ist der Mensch von Natur aus organisch mittellos, instinktlos und auf sich selbst gestellt, seine Existenz ist "sein eigenes Werk". Beide Philosophen gehen zwar von demselben Punkt aus, aber sie ziehen daraus verschiedene Schlüsse. In der kantischen Philosophie geht es vor allem um die Erwerbung vernünftiger Moralität - der Mensch soll aus seiner selbstverschuldeten Unmündlichkeit ausgehen und als rationales Wesen moralische Reife erreichen,

29 J.G. Herder, Abhandlung..., op. cit., S. 25.

30 Ibidem.

31 Ibidem, S. 28. 
Herder betont dagegen, dass das Ziel des menschlichen Lebens die Ausbildung der Humanität ist, also der Mensch soll alle psycho-physische, sowohl emotionelle, als auch intellektuelle Fähigkeiten entwickeln, weil die menschliche Vernuft, Freiheit und Moralität im Grunde genommen auf der Leiblichkeit gegründet sind und aller Fortschritt sich vermittelst der Sprache vollzieht. Es gibt kein Denken ohne Sprache und keine Sprache ohne Sinnlichkeit.

Kant interessiert sich überhaupt nicht für die Sprachentwicklung, weil er die Existenz der universellen, ahistorischen Vernunft voraussetzt. Für Herder, solche ganz unkritische Voraussetzung ist selbst beweispflichtig. Die Sprachvergessenheit macht zweifelsohne einen blinden Fleck im kantischen System, den Herder immer wieder verbissen und vehement kritisiert. Er betrachtet Kants kritische Philosophie als eine Art von unbegründeter Metaphysik. Nachdem Kant die Herkunft der menschlichen Vernunft aus dem Bereich der Sinnlichkeit ignoriert hatte, ließ er sich dazu verführen, bloße „Wortformeln” und trügerische transzendentale „Idolen” zu produzieren, die das natürliche Potential der Subjektivität verdecken.

Für Herder, die menschliche Vernunft konzentriert sich ausschließlich auf ihre natürliche Disposition und versteht Wirklichkeit als unendliche Fülle sinnlich erfahrbarer Kraft, die in ihrer Mannigfaltigkeit harmonisch zu einer Einheit zusammenstimmt. Zu diesem Zweck muß auf ein transzendental-begriffliches System der Wirklichkeitsinterpretation verzichtet werden, weil es auf der irrigen Voraussetzung beruht, daß die menschliche Subjektivität als eine nur eigengesetzlich bestimmbare Instanz als autonome Interpretin der Natur gegenübertritt und deshalb legitimiert ist. Es ist ganz falsch die Wirklichkeit nach Maßgabe ihrer von der Natur unabhängigen, apriorischen Struktur auszulegen. Für Herder entscheidend ist vielmehr, daß die Erfahrung der Natur durch eine unbeschränkte, aber nicht ganz amorphe Vielfalt einzelner sinnlicher Impressionen begründet wird. Diese werden wiederum durch die bilderschaffende Einbildungskraft und die Sprache strukturiert. Wegen des Verlustes unmittelbarer Identität mit der Natur unterliegen aber menschliche Vernunft und Freiheit der Gefahr ihrer Verselbständigung. Die Einsicht in die Gesetzmäßigkeit der Sprache soll die Begründung einer Metaphysik verhindern, die positiv auf der Differenz von Subjektivität und Natur aufbaut. Sprache als das erste Produkt der menschlichen Freiheit beruht dennoch auf einer Gesetzmäßigkeit der Natur.

Die Einsicht in die naturanaloge Regelhaftigkeit der Sprache legt es nahe, die Differenz von Natur und Subjektivität lediglich als den negativen Fall menschlicher Selbstentfremdung zu begreifen. Im Gegensatz zur Kantischen Betonung der Autonomie der transzendentalphilosophisch begründeten Subjektivität wird Sprache zum Paradigma aller der Betätigungen des Menschen, die ihn an das überlegene Kraftpotential 
der Natur zurückbinden. Kant auf irrige Weise fasst der Sprache als technisches Instrumentarium des Denkens auf. Für Herder resultiert aus diesem Verfahren ein Verlust des dem Menschen unverzichtbaren Anspruchs auf Anschaulichkeit seiner Erkenntnisse, weil die Fachsprache der Schulphilosophie lediglich auf der "Wirkung der Abstraktion” beruht. Indem sie im Abstraktionsvorgang die natürlich-sinnliche Wirklichkeit ausschaltet, konstruiert sie lediglich einen "Scheinbegriff”, dessen Bedeutung intersubjektiv nicht mehr verstanden werden kann.

In der kritischen Distanzierung von Kant spricht Herder der produktiven Einbildungskraft und ihrem poetischen Potential die Funktion der einheitlichen Grundkraft der Subjektivität zu. Deren entscheidende Leistung ist die autonome Produktion von „Bildern”, die eine primär diffuse Wirklichkeit dem menschlichen Bewußtsein verständlich machen. Die Koordination dieser sinnlichen also nicht nur optischen, sondern auch taktilen und vor allem akustischen Bilder leistet die Sprache. Allein in diesem Akt autonomer Sinnproduktion konzentrieren sich die unterschiedlichen Vermögen der Seele in einer einzigen Kraft. Herder beschreibt diesen Prozess in seiner Abhandlung über den Ursprung der Sprache.

\section{Besonnenheit und die Genese der Sprache als Energeia}

In der Abhandlung über den Ursprung der Sprache setzt sich Herder zum Ziel, die Sprachartigkeit von Natur, Geist und Geschichte und die Teilnahme des Menschen an ihnen darzulegen. Ihre zwei Teile besprachen die Möglichkeit, aus den Fähigkeiten des Menschen Sprache zu finden und zu bilden, sowie die Gesetze der geschichtsbdenden Wechselwirkung der Sprachfähigkeit imt der kulturgeographischen und soziologischen "Sphäre” des Menschen. Herder scheint, zu sagen, über den Ursprung der Sprache läßt sich nichts denken und nichts sagen, da dies - aufgrund der Wechelbeziehung von Sprache und Denken - ein logisch widersprüchlicher Selbstbegründungsversuch des Denkens wäre.

Der Philosoph beginnt seine Argumentation von der berühmten Äußerung, "schon als Tier hat der Mensch Sprache." ${ }^{32}$ Es ist noch nicht die Rede im strengen Sinne, aber die spontane "Mechanik fühlender Körper." 33 Sowohl menschlichen, als auch tierischen Organismen zeigen eine angeborene Neigung, die emotional relevanten Empfindungen zu artikulieren. Auf diese Weise betont Herder die Gemeinschaft des Men-

\footnotetext{
32 Ibidem, S. 4.

33 Ibidem.
} 
schen mit der Tierwelt und mit der gesamten Natur. Die Natur wird betrachtet von ihm als einen organischen Zusammenhang, in dem alle Lebewesen sich miteinander kommunizieren. Im Fall der höher entwikkelten Tiere nimmt diese Kommunikation die Form einer "Sprache der Empfindung", die der menschlichen Sprache zugrundeliegt. Die expressive Artikulationen „,sind nicht die eigentlichen Wurzeln, aber die Säfte, die die Wurzeln der Sprache beleben." ${ }^{34}$ Reste dieser expressiven Naturtöne werden noch in ältesten Sprachen bewahrt, aber sie erfüllen keine kommunikative Funktion im engeren Sinne des Wortes, es fehlt ihnen eine reflexive Intentionalität, sie drücken nur innere, emotionelle $\mathrm{Zu}$ stände aus. Die menschliche Rede ist dagegen von Natur aus intentional.

Tierlaute sind sprachartig. Der Naturschrei des tierischen Mechanismus .B. als Anzeichen von Schmerz ist allen stimmbegbten Tieren gemeinsam und wird artunabhängig gegört; die Tiergattungen haben ihre arteigenen Verständigungssysteme, die bei Haustieren der Mench zu verstehen und zu nutzen sucht; im Seufzen, Hauchen, Stöhnen, mit dem auch die Tiere Aufmerksamkeit und Rührung anderer Tiere und des Menschen zu wecken suchen und das in Ursprachen besonders stark ist, atmet gleichsam der allverbindende Lebensgeist. Über diese drei Ursprünge verfügt seiner tierischen Leibesverfassheit gemäß auch der Mensch. Naturlaute werden in kindlichen Sprachen nachgeahmt, aber „zu Merkmale vom Verstande geprägt” ${ }^{35}$ und „innerhalb der natürlichen Tonleiter der menschlichen Stimme." ${ }^{36}$ Höher entwickelte Sprachen überlagern diese Anfänge durch ein Ganzes aus System und Handlung, ergon und energeia - „Besonnenheit und Sprache, Besinnung und Sprachschaffung ${ }^{\prime \prime 7}$ in dem das Sprachartige von Natur und Geist im Menschen, seinem Sprachbesitz und seiner Sprachbildung, zusammenwirken und die reale, sich mehr und mehr differenzierende Sprache konstituieren.

Im Gegensatz zu Tieren verfügt aber der Mensch über eine in der Tierwelt einamaliges Vermögen - die Besonneheit oder - wie Herder sie auch nennt - die Reflexion, durch die er sich auf die Aktivität eigener Sinne beziehen kann und aus dem ,[...] ganzen Ozean von Empfindungen, der sie durch, alle Sinnen durchrauschet, eine Welle absondern, sie anhalten, die Aufmerksamkeit auf sie richten." ${ }^{38}$ Dieses Vermögen ist im Menschen von Anbeginn an - dh. in der philo- und ontogenetischen Perspektive - immer tätig. Die Besonneheit, die „[...] keine abgeteilte, einzelnwürkende Kraft, sondern eine seiner Gattung eigne Richtung aller

\footnotetext{
${ }^{34}$ Ibidem, S. 8-9.

35 Ibidem, S. 70.

36 Ibidem, S. 69.

37 Ibidem, S. 82.

38 Ibidem, S. 40.
} 
Kräfte" ${ }^{\prime 39}$ ist, bildet somit eine Art von der energetischen Konfiguration der sinnlichen, affektiv gefärbten Impulse und Triebe. Es gibt im Menschen kein einzelnes Vermögen wie Instinkt, Einbildungskraft, Verstand, Sinnlichkeit oder Begehrungsvermögen - sie alle sind - wie schreibt Herder - nur Bestimmungen einer einzigen Kraft oder „,[...] die gänzliche Bestimmung seiner denkenden Kraft im Verhältnis seiner Sinnlichkeit und Triebe." 40

Der Philosoph konzipiert ein holistisches und energetisches Bild der Seele, die ein integraler Teil der Natur Ist. Die an sich flüssige Natur bildet sich nach dem Gesetz der Krafterhaltung stets „zur Kugelform." ${ }^{41}$ Diese Aussage gilt für alle Planeten- und Sonnensysteme. Auch in seiner Gesamtheit ist der Kosmos „[...] eine Kugel, die auf sich selbst ruhet." 42 Die Metaphorik der Kugel beschreibt ein Verfahren der Selbsterhaltung durch Selbstbezüglichkeit, das für alle naturhaften Prozesse gültig ist. Demnach erhalten alle natürlichen Kräfte ihr energetisches Potential dadurch, daß sie beständig auf ihren eigenen Ausgangspunkt zurückwirken. Auch die menschliche Subjektivität funktioniert als ein solches homoestatisches System. Deshalb Herder behauptet, dass allein diejenigen Begriffsbildungen der menschlichen Vernunft deren naturanaloge Gesetzmäßigkeit respektieren, die sozusagen aus Vielem, das uns zuströmt, ein „lichtes Eins" machen. Ausschließlich in dieser komplexitätsreduzierenden, anschauungserhaltenden und handlungsermöglichenden Funktion vernimmt die menschliche Vernunft "eine Art Rückwirkung” auf sich selbst, durch die sie "am hellesten fühlet, daß sie Eins, ein Selbst ist." ${ }^{43}$ Nur in dieser Bestimmung greift das menschliche Denken nicht über sich selbst hinaus, sondern gewinnt gleichsam seinen verlorenen archimedischen Punkt zurück. ${ }^{44}$

Die Vereinheitlichung der menschlichen Erfahrung ist nicht die Folge der begrifflichen Spontanäitet - wie Kant meint - sondern sie resultiert aus der Reflexion, die entsprechende Triebimpulse, sinnliche Vorstellungen und sprachliche Zeichen dem homoestatischen Gesetz gemäß koordiniert und in die Handlung umsetzt.

Für Herder ist der Nervenreiz die Basis der einfachsten sinnlichen Empfindungen, die bis zur Einbildungskraft transportiert werden müssen. In ihrer vermittelnden Funktion nennt Herder sie „zarte Silberbande, dadurch der Schöpfer die innere und äußere Welt [...] knüpfet." ${ }^{45}$

39 Ibidem, S. 36.

40 Ibidem, S. 34.

${ }^{41}$ J.G. Herder, J.G. Herder, Von der Lebensarten der Sprache ..., op. cit. S. 153.

42 Ibidem, S. 554.

43 J.G. Herder, Vom Erkennen und Empfinden der menschlichen Seele [in:] Herders Sämmtliche Werke. (hrs. von B. Suphan), 8. Band, Berlin 1892, S. 193.

44 Ibidem, S. 195.

45 Ibidem, S. 190. 
Diese organisiert dagegen die Sinnesempfindungen als sinnliche Bilder, die wiederum durch Sprache strukturiert werden. Das produktive Energiezentrum des Menschen funktioniert nach der Gesetzmäßigkeit der Reiz- und Empfindungsübertragung. Dieses in einem Nervensystem vewurfzelten Zentrum organisiert alle Aktionen des menschlichen Denkens und Handelns zu einer Einheit. ${ }^{46}$

Die Subjektivität beruht auf der Wahrnehmung seiner selbst und der Wahrnehmung von Wirklichkeit. Herder deutet sie als einen physikalischen Prozeß der Energieaufnahme und der Energieabfuhr. Die dem Subjekt von der Natur zugeführte Energie muß wieder an die Natur oder an andere Menschen durch versprachlichte Handlung „abgeführt” werden. Nur wenn dieser Energieaustauschprozeß ohne hemmende Reibung abläuft, bilden theoretische und praktische Vernunft des Menschen eine unzertrennliche Einheit, in der sich der Mensch in seinem substantiellen Kern als die Einheit eines energetisch produzierenden Subjekts erfährt.

Die Besonnenheit, die eine unstabile Sinnlichkeit koordiniert, ermöglicht uns, den Abstand gegenüber der Umwelt und den internen Prozessen zu erhalten. Einzelne Sinne sind keine geschlossenen, isolierten Sinnesfelder, sondern sie sich miteinander reibungslos kommunizieren und zusammenarbeiten. Eindrücke einer Sinne können in die "Sprache” anderer Sinne umgesetzt werden und dadurch gewinnt menschliche Wahrnehmung symbolische Qualitäten. Zum Beispiel, ein optischer Effekt der schillernden Oberfläche wird zu einem Symbol für ihre Glattheit. „Wie hängt Gesicht und Gehör, Farbe und Wort, Duft und Ton zusammen? - fragt Herder und sofort beantwortet seine Frage - Nicht unter sich in den Gegenständen; aber was sind denn diese Eigenschaften in den Gegenständen? Sie sind bloß sinnliche Empfindungen in uns, und als solche, fließen sie nicht alle in eins? Wir sind ein denkendes sensorium commune, nur von verschiednen Seiten berührt - da liegt die Erklärung." ${ }^{\prime 7}$ Die Fähigkeit, Eindrücke aufeinander zu transponieren organisiert die Aktivität der Sinne noch auf der vorsprachlichen Ettape der Entwicklung, aber erst die akustische Rede ermöglicht die vielseitige typisch menschliche Kommunkation zwischen verschieden Sinnen. Das Gehör - der wichtigste aller Sinne - wird gleichsam dafür prädestiniert, die führende Rolle in diesem Prozess zu spielen.

Erstens, die akustischen Eindrücke sind durch die moderate Empfindbarkeit gekennzeichnet, deswegen können sie einfach im Wahrnehmungsstrom festgehalten werden. Das Gehör führt (durch die Töne) die Umwelt zum Inneren des Menschen und wird dadurch zu einem natürlichen Medium für die Synthese aller Sinne. „Die Empfindungen verei-

46 Ibidem, S. 191.

47 J.G. Herder, Abhandlung ..., op. cit., S. 71. 
nigen sich - schreibt Herder - und kommen also alle der Gegend nahe, wo Merkmale zu Schällen werden. So wird, was man sieht, so wird, was man fühlt, auch tönbar." 48 Für Herder ist der Schluss daraus selbstverständlich: „Der Sinn zur Sprache ist unser Mittel und Vereinigungssinn geworden; wir sind Sprachgeschöpfe." 49

Zweitens, das Gehör ist ein mittlerer Sinn in Bezug auf die Klarheit und Deutlichkeit der rezipierten Merkmale. Die taktilen Eindrücke sind zu unbestimmt, während die optischen Daten erdrücken uns mit dem Überschuss der Eigenschaften. Das Gehör führt dagegen moderate Einheitlichkeit in unsere Sinnlichkeit ein. Es ist auch ein mittlerer Sinn in Ansehung der Lebhaftigkeit oder der Intensivität der Eindrücke. Das Sehvermögen ist gleichsam kalt, gleichgültig für die Welt, es distanziert sich von der wahrgenommenen Objekte, versucht sie optisch zu beherschen. Der Tastsinn wirkt am meisten auf einen relativ kleinen Bereich unseres Körpers, er angagiert und absorbiert uns sehr stark. Die Töne erfüllen uns, aber sie stören nicht andere Sinne. Diese synthetisierende Funktion des Gehörs hängt mit seiner Temporalität zusammen. Es stellt die Welt als zeitliche Nachfolge der Bilder dar, die durch verschiedene Sinne geformt werden und zugleich ermöglicht uns Distanz zu inneren Vorstellungen zu wahren. Das Sehvermögen fasst die Wirklichkeit simultan in einem Augenblick, und der Tastsinn beschränkt sich nur auf einen Moment der Empfindung. Viertens, das Gehör eignet sich am besten für die Expression menschlicher Bedürfnisse, der Tastsinn wirkt eigennützig und ist in sich gesenkt, der Gesichtssinn ist dagegen stumm und ineffektiv in der Kommunikation. Endlich, das Gehör zeichnet sich in Anbetracht seiner Entwicklung aus. Seine Artikulationsfähigkeiten sind sehr plastisch und deswegen müssen sie stufenweise entwickelt werden. Davon profitiert auch das Sehvermögen, weil die Sprachentwicklung, die mit der Entwicklung des Denkens verbunden ist, beeinlusst die Selektionsprozesse im Sehfeld. Die privilegierte Stellung des Gehörs unter den anderen Sinnen erklärt aber nicht vollumfänglich den Ursprung der Sprache.

Herder ergänzt die Deduktion der Sprache aus de qualitätiven Eigenschaften der Sinne, indem er auf die allgemeinen Gesetze der menschlichen Entwicklung hinweist. Das wichtigste Gesetz ist das Prinzip der "Ökonomie der Natur", wonach der Mensch als Mängelwesen seine biologische Defizite mit Hilfe seiner Vernunft und Sprache kompensiert. Sprache fungiert als unverzichtbares Werkzeug in dem Prozess der Artikulation und Organisation menschlicher Bedürfnisse. Sie ist auch ein ideales Medium, das die menschliche Erfahrung akkumuliert und den Menschen bei der Beherrschung der Natur stützt. Die Geschichte ist

48 Ibidem, S. 77.

49 Ibidem. 
konstituiert durch die Entwicklung und Tradition von Sprachen, mithin von Denken und Kultur. Der geschichtlich sich aus primitiven Anfängen in steigenden Sprachentwicklung muss eine Entwicklung im Denken entsprechen, die ihrerseits jede der Stationen ihres Progresses vom Primitiven zum Differenziert-Komplexen als Zeichen für das Ganze einer tatsächlich unabschließbaren Erkenntnis zu betrachten nötigt und damit sprachartig ist.

Sprachphilosophische, sprachwissenschaftliche und sprachgeschichtliche Reflexion bleibt noch ungetrennt bei Herder und durchzieht nicht nur, sondern begründet sein Werk, nimmt die Tradition auf, transformiert sie unter der anthropologischen Prämisse und denkt dem 20. Jahrhundert in einer Weise vor, die erst jetzt erkannt werden kann.

\section{Bibliography}

Blumenberg, H. Paradigmen zur Metaphorologie, Bonn 1960.

Descartes, R. Abhandlung über die Methode [in:] idem, Philosophische Schriften in einem Band, Hamburg 1996.

Gadamer, H.-G. Wahrheit und Methode, Tübingen, 1965.

Herder, J.G. Abhandlung über den Ursprung der Sprache, Stuttgart 1986.

Herder, J.G. Vom Erkennen und Empfinden der menschlichen Seele [in:] Herders Sämmtliche Werke. (hrs. von B. Suphan), 8. Band, Berlin 1892.

Herder, J.G. Von der Lebensarten der Sprache [in:] Herders Sämmtliche Werke. (hrs. von B. Suphan), 1. Band, Berlin 1877.

Herder, J.G. Wie klebt in der Weltweisheit der Gedanke am Ausdruck, sinnlich, technisch oder grammatisch? [in:] Herders Sämmtliche Werke. (hrs. von B. Suphan), 1. Band, Berlin 1877.

Herder, J.G. Eine Metakritik zur Kritik der reinen Vernunft [in:] „Schriften zur Literatur und Philosophie, 1792-1800" (hrS. von H.D. Irmscher), Frankfurt am Main 1998.

Humboldt, W. Schriften zur Sprachphilosophie. Darmstadt 1963.

Kant, I. Kritik der reinen Vernunft, Hamburg 1967.

Kant, I. Recensionen von J.G.Herders Ideen zur Philosophie der Geschichte der Menschheit. Theil 1. 2. [in:] Kant's Gesammelte Schriften „Akademieausgabe", Königlich Preußische Akademie der Wissenschaften, B. 8, Berlin 1923.

Kant, I. Anthropologie in pragmatischer Hinsicht [in:] Kant's Gesammelte Schriften "Akademieausgabe“, Königlich Preußische Akademie der Wissenschaften, B. 7, Berlin 1917.

Kant, I. Prolegomena zu einer jeden künftigen Metaphysik, die als Wissenschaft wird auftreten können [in:] Kant's Gesammelte Schriften "Akademieausgabe“, Königlich Preußische Akademie der Wissenschaften, B. 4, Berlin 1911. 
Kant, I. Untersuchung über die Deutlichkeit der Grundsätze der natürlichen Theologie und der Moral [in:] Kant's Gesammelte Schriften "Akademieausgabe", Königlich Preußische Akademie der Wissenschaften, B. 2, Berlin 1912.

Kant, I. Träume eines Geistersehers, erläutert durch Träume der Metaphysik [in:] Kant's Gesammelte Schriften "Akademieausgabe“, Königlich Preußische Akademie der Wissenschaften, B. 2, Berlin 1912.

Kant, I. Kritik der Urteilskraft, Hamburg 2002.

\section{Summary}

The aim of the paper is to compare the concept of language in the philosophy of Herder and Kant. Kant insists on the dualism of thought and language - concepts, thoughts and ideas are ontologically preverbal. In this regard, the linguistic sign is just a connection medium that connects the idea with the object and guarantees the intersubjective communication. The linguistic signs are nothing of importance for objective knowledge. Kant knows that in any empirical language works an anonymous form of world interpretation and it precedes all philosophical reflection. This understanding of reality in everyday speech use "works" with sufficient precision, but it is impossible to deduce the whole system of categories from the analysis of language. According to Herder all knowledge is founded on the basis of sensory experience and subjected to historical development.All terms are shaped by sensual imagination, there are no pure categories that would not mediated by senses. The conclusion of its deliberations is the thesis: "no thought without language, no language without sensuality." Man is a deficient being. He is not adapted to any environment, he has no specialized instincts and sensory organs, and in this sense he is world-open being. However, this unfavorable initial situation has a functional sense. The human deficites are compensated by the language that opens up an absent in the animal kingdom, almost unlimited perspective of the freedom.

\section{Keywords}

language, intersubjective communication, interpretation, instincts, concept, environment 